Recherches en didactique des langues et des cultures

Les cahiers de l'Acedle

17-2 | 2020

Recherches collaboratives en didactique des langues

\title{
Dynamique d'apprenance dans une recherche collaborative portant sur la didactique de l'oral en contexte plurilingue : regards croisés
}

Véronique Miguel-Addisu et Sophie Beaumont

\section{OpenEdition \\ 1 Journals}

Édition électronique

URL : https://journals.openedition.org/rdlc/7371

DOI : 10.4000/rdlc.7371

ISSN : 1958-5772

Éditeur

ACEDLE

Référence électronique

Véronique Miguel-Addisu et Sophie Beaumont, « Dynamique d'apprenance dans une recherche collaborative portant sur la didactique de l'oral en contexte plurilingue : regards croisés », Recherches en didactique des langues et des cultures [En ligne], 17-2 | 2020, mis en ligne le 27 avril 2020, consulté le 17 octobre 2022. URL : http://journals.openedition.org/rdlc/7371 ; DOI : https://doi.org/10.4000/rdlc. 7371

Ce document a été généré automatiquement le 17 octobre 2022

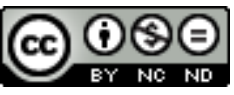

Creative Commons - Attribution - Pas d'Utilisation Commerciale - Pas de Modification 4.0 International - CC BY-NC-ND 4.0

https://creativecommons.org/licenses/by-nc-nd/4.0/ 


\title{
Dynamique d'apprenance dans une recherche collaborative portant sur la didactique de l'oral en contexte plurilingue : regards croisés
}

\author{
Véronique Miguel-Addisu et Sophie Beaumont
}

1 Si on peut décrire les démarches propres aux recherches collaboratives (désormais RC) d'un point de vue scientifique, nous postulons ici que le croisement de regards des différents collaborateurs peut nous apprendre beaucoup sur la dynamique qu'elle suppose et sur les effets qu'elle produit. Cette question nous intéresse parce que dans le champ de la recherche-formation que nous explorons, les résultats attendus par les praticiens ne sont pas du même ordre que ceux poursuivis par les chercheurs (Desgagné et al., 2001; Morrissette, 2013). Pourtant, la collaboration est possible. Comment? Peut-on en identifier des leviers qui ne soient pas uniquement de l'ordre du relationnel, de «l'heureuse rencontre », ou même d'une éthique partagée qui définirait plus spécifiquement les RC (Anadon, 2013)?

2 Cette question sera explorée ici à travers le projet École, Parole de l'élève et Plurilinguisme : Recherche Collaborative au lycée français de San Francisco - AEFE, USA (désormais E2PRC_Francisco), qui se terminera fin 2020 après deux années de collaboration.

3 À l'origine du projet, il y a le besoin d'un établissement sur une question didactique : comment améliorer l'enseignement de l'oral dans la langue de scolarisation (le français) en contexte anglophone (aux États-Unis), et un besoin scientifique de documentation des pratiques et représentations langagières des acteurs de l'enseignement/apprentissage en situation immersive (élèves, enseignants). Le projet comporte deux volets auxquels tous les acteurs ont participé: une enquête sociolinguistique initiée par les chercheurs et soutenue par les praticiens (questionnaires, entretiens et observations de classe), et une démarche de formation didactique initiée par les formateurs et soutenue par les chercheurs (analyse de pratiques outillées par la recherche, expérimentations, productions pédagogiques). Le 
postulat sociodidactique, partagé par les praticiens et les chercheurs, est que ces deux volets sont complémentaires: les praticiens ont besoin de mieux comprendre le contexte sociolinguistique pour soutenir l'appropriation de leurs élèves, les chercheurs ont besoin de comprendre comment les praticiens tirent parti de ces connaissances en termes didactiques. Le postulat partagé par tous est que leur alliance est possible, et souhaitable, au bénéfice de tous les élèves. Le projet a donc une visée inclusive.

Nous nous intéresserons ici plus spécifiquement à l'analyse du processus de collaboration entre chercheurs et formateurs par les deux voix des co-pilotes du projet. Sophie Beaumont, inspectrice de l'éducation nationale AEFE pour la zone Amérique du nord (désormais IEN $)^{1}$, s'intéresse ici principalement aux processus d'apprenance mis en place avec les douze praticiens pendant les deux années du projet ${ }^{2}$, avec l'appui des deux formatrices qui accompagnent plus directement l'équipe enseignante (Elsa Rodriguez, co-directrice de l'école primaire, désormais DIR; Laurence Madamour, conseillère pédagogique adjointe à l'IEN pour la zone Ouest des États-Unis et du Canada, désormais CPAIEN). Véronique Miguel Addisu, chercheure à l'université de Rouen et formatrice à l'INSPE (désormais EC) explicite l'implication des chercheurs et l'intérêt scientifique de cette collaboration pour la didactique de l'oral, du français, et du plurilinguisme, avec l'appui de l'équipe de chercheurs membres du projet (Mehmet-Ali Akinci, acquisition bilingue ; Evelyne Delabarre, didactique de l'oral ; Christel Troncy, didactique des langues) $)^{3}$.

Après avoir présenté le contexte de la recherche collaborative E2PRC_Francisco dans le champ des recherches collaboratives et de la didactique de l'oral en contexte plurilingue, nous nous arrêterons ${ }^{4}$ sur son impact en termes de formation et d'apprenance et montrerons en quoi la recherche y a contribué, et s'en est nourrie. Puis nous traiterons des transformations et déplacements professionnels qu'un tel dispositif suppose pour les formateurs et les deux co-pilotes engagées.

\section{Naissance d'un projet collaboratif : contexte, apprenance et problématisation de la didactique de l'oral en contexte plurilingue}

\section{Un contexte scolaire français à l'étranger : l'AEFE}

6 L'agence pour l'enseignement du français à l'étranger (désormais AEFE) constitue un réseau de plus de 500 écoles qui enseignent les programmes français dans près de 140 pays dans le monde. Ces établissements s'engagent à respecter les valeurs de l'éducation nationale française et sa structure pédagogique. La zone AEFE « Amérique du nord » scolarise près de 25000 élèves dont $64 \%$ ont entre 3 et 11 ans.

7 Alors qu'une des missions de l'AEFE est de proposer une continuité de la scolarité sur programmes français aux familles expatriées, la présence des élèves de nationalité française et au profil linguistique monolingue est variable et peut être minoritaire dans de nombreuses écoles. La plupart des familles parle d'autres langues à la maison, et le plus souvent la langue du pays hôte. C'est ici le cas. Le choix de la scolarité en français n'est pas leur seule motivation : la réputation d'excellence des établissements est aussi la raison pour laquelle les familles privilégient ces écoles, souvent privées. 
8 De nombreuses recherches montrent que bien menée, une politique d'éducation plurilingue harmonieuse favorise le développement sociocognitif de tous les élèves, et une société plus démocratique (Nussbaum, 2014; Cummins, 2014). Deux priorités complémentaires sont identifiées par l'AEFE pour ce qui concerne la didactique des langues: assurer une bonne maitrise du français, qui doit être la langue de scolarité dominante, et adopter une approche plurilingue valorisant les langues et cultures $\mathrm{du}$ pays d'accueil et des familles (AEFE, 2015). Or cette complémentarité des langues reste un défi dans des écoles du réseau qui, en diffusant «l'enseignement français » à l'étranger, restent de tradition monolingue.

9 L'école primaire SF1, est représentative d'un établissement français à l'étranger dans sa structure, ses missions et son public. C'est une école privée américaine, homologuée par la France, qui adopte le curriculum français de la maternelle à la terminale, année $\mathrm{du}$ baccalauréat. Elle est pilotée par une équipe de direction française expatriée (proviseur, proviseur adjoint, deux directeurs du primaire) et accompagnée par l'équipe de zone, (CPAIEN et IEN) également expatriés. Deux formatrices et neuf enseignants volontaires intervenant sur des niveaux de la PS au CM2 se sont engagés dans la démarche réflexive l'année 1. Tous les enseignants impliqués sont français et expérimentés dans le réseau AEFE, où ils enseignent depuis plusieurs années.

Les classes suivent le programme du ministère de l'éducation nationale français: quelles que soient leurs langues familiales, les élèves apprennent dans un cadre d'immersion en français, avec en majorité des enseignants français titulaires de l'éducation nationale. Cependant, l'enseignement de l'anglais et en anglais est renforcé (8h hebdomadaires) et assuré par des enseignants américains, eux-mêmes souvent non francophones. Ils n'ont pas eu l'opportunité de participer à cette recherche. Dans cette école, les élèves anglophones qui découvrent et apprennent la langue française principalement au travers de leur scolarité sont les plus nombreux dans les classes.

\section{Genèse d'une réflexivité conjointe sur la didactique de l'oral dans un établissement français typique du réseau $\mathrm{AEFE}$}

11 L'année précédant le projet, IEN a identifié un besoin de formation spécifique pour l'établissement SF1: construire une didactique de l'oral répondant mieux aux besoins de ces élèves grandissant dans un contexte plurilingue. Les enseignants français soulignaient en effet qu'ils percevaient la richesse de ce plurilinguisme mais qu'ils ne savaient comment faire pour s'y appuyer à des fins didactiques, en particulier à l'oral : dans la classe, anglais et français alternent quotidiennement dans les paroles des élèves, qui parfois ne savent pas eux-mêmes comment gérer les passages d'une langue à une autre dans les interactions. Certains étaient gênés par le plurilinguisme ambiant mais voulaient dépasser ce ressenti et aspiraient à un questionnement réflexif sur cette situation. IEN a alors sollicité une équipe de chercheurs s'intéressant aux interactions didactiques en contexte plurilingue et susceptibles de s'engager dans un processus de recherche-formation pour répondre à un objectif de développement professionnel. Le but était de trouver ensemble des "solutions» que peut-être les praticiens ne connaissaient pas.

12 Dès le début du projet, une explicitation du rôle de la recherche a été utile pour éviter une vision applicationniste de la recherche-formation collaborative, démarche avant tout réflexive, surtout en didactique : 
[Le chercheur en didactique des langues] a pour rôle de proposer un certain nombre de réflexions, voire de pistes, situées et argumentées, susceptibles de donner aux praticiens les moyens de faire des choix motivés et de construire leur activité professionnelle en s'appuyant sur les réflexions engagées, les arguments avancés, les critiques formulées, les débats en cours (Castellotti, 2013 : 77-78). par exemple: Lafontaine, 2013) mais pour leurs analyses, ils se sont principalement inspirés des travaux scientifiques portant sur les interactions en classe de langue (Cicurel, 2011) et en classe de français (Nonnon, 2011). Ils souhaitaient faire comprendre que la didactique de l'oral est un «objet verbal non identifié » (Halté, 2005), qui a une histoire scolaire située (Pégaz-Paquet et Cadet, 2016), que l'on appréhende différemment selon que l'on se réfère aux genres discursifs (Dolz et Schneuwly, 1998), ou aux ressources interactionnelles plurilingues (Nussbaum, 2014). L'objet de la recherche soulevait nombre de questions vives en didactique (du français, des langues), que les chercheurs entendaient faire émerger avec les praticiens pour qu'une réflexivité partagée puisse s'initier :

Plus que toute autre, la didactique de l'oral s'appuie sur des pratiques et des actions fortement contextualisées, mettant directement en jeu les acteurs selon un degré d'implication fort, des scénarios non complètement prévisibles (malgré la ritualisation de la communication scolaire), et sur un matériau émergent, qui relève en partie de l'événement, au-delà des grandes régularités liées à la forme scolaire. Comme on l'a vu, les modèles à priori, les règles générales qui en seraient déduites sont faiblement opératoires pour orienter les conduites en situation, si ces règles ne sont pas actualisées concrètement et spécifiquement par rapport aux expériences partagées (...). (Nonnon, 2011 : 205)

D'autres pistes pédagogiques ont été proposées par les chercheurs pour l'analyse du geste professionnel et l'enseignement de l'oral ; elles ont été mutualisées sur un padlet commun ${ }^{5}$. Après la phase de problématisation menée par les chercheurs, une formation pédagogique s'est avérée nécessaire pour clarifier la notion de didactique de l'oral pour les praticiens, elle a été menée par la formatrice CPAIEN en décembre de l'année 1, qui s'est appuyée sur les programmes officiels, et a montré la pertinence de la prise en compte du plurilinguisme des élèves à cet effet :

Figure 1 : trois entrées pour la didactique de l'oral (source : formation CPAIEN, décembre 2018)

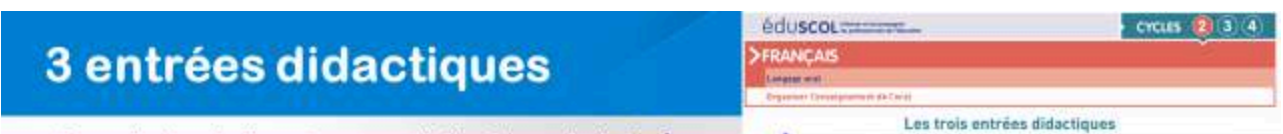

L'organisation de l'enseignement de l'oral à partir de trois approches :

L’approche communicationnelle : l'école et la classe comme micro sociétés, l'oral comme moyen d'expression et de communication respect des règles de communication compréhension des propos (en émission, en réception)

L'approche discursive : la classe comme lieu de construction des savoirs, I'oral pour apprendre les conduites discursives dans les disciplines : raconter, décrire, expliquer, justifier, argumenter, prescrire

L'approche intégrée : I'oral comme objet d'apprentissage,

l'oral à apprendre

le récit

l'exposé

le débat délibératif ou interprétatif

la mise en voix de textes,... 


\section{L'apprenance : une dynamique pertinente pour s'intéresser aux plurilinguismes des élèves}

15 Fondé sur le croisement des interprétations touchant aux pratiques de classes ainsi qu'aux pratiques langagières des élèves, le protocole de la RC prend appui sur la réflexivité de tous les acteurs :

16 Praticiens et chercheurs sont amenés à interagir et à explorer ensemble un aspect de la pratique d'un intérêt commun. Cette activité réflexive s'appuie essentiellement sur l'explicitation et l'analyse de situations de pratique vécues par les enseignants, sous l'angle de l'intérêt commun défini par le projet d'exploration. C'est dire que l'activité est aménagée de telle sorte qu'elle favorise et fait en sorte que soit entretenue une sorte de "conversation", pour emprunter à Schön (1984), entre la pratique (des enseignants) et le retour réflexif sur cette pratique (entre praticiens et chercheurs). (Desgagné et al. $2001: 37$ ).

17 Le processus de contextualisation mobilise altérité et réflexivité sur un temps long, et s'appuie sur une démarche d'apprenance individuelle et collective, définie comme « une attitude propice à l'acte d'apprendre dans toutes les situations, qu'elles soient formelles ou non, expérientielles ou didactiques, autodirigées ou dirigées, intentionnelles ou fortuites » (Carré, 2005 : 109). Cette dynamique est établie lorsque les membres sont acteurs de leur développement professionnel (engagement pratique), lorsqu'ils ressentent que le projet est le leur, et qu'ils transforment effectivement leurs pratiques (engagement affectif), lorsqu'ils orientent leurs choix pratiques en vue d'apprendre (engagement conatif). Sont concernés les enseignants comme les formateurs, et les chercheurs: tous pensent leur action en la réalisant, ils la construisent, l'improvisent en partie, innovent, recadrent leurs problèmes en tachant de leur donner du sens et agissent de manière souvent créatrice pour les résoudre, ils considèrent que le regard des autres peut les aider. Leur réflexivité s'appuie sur des savoirs scientifiques permettant d'engager des analyses interprétatives et des choix praxéologiques.

Figure 2 : la dynamique d'apprenance dans le projet E2PRC_Francisco

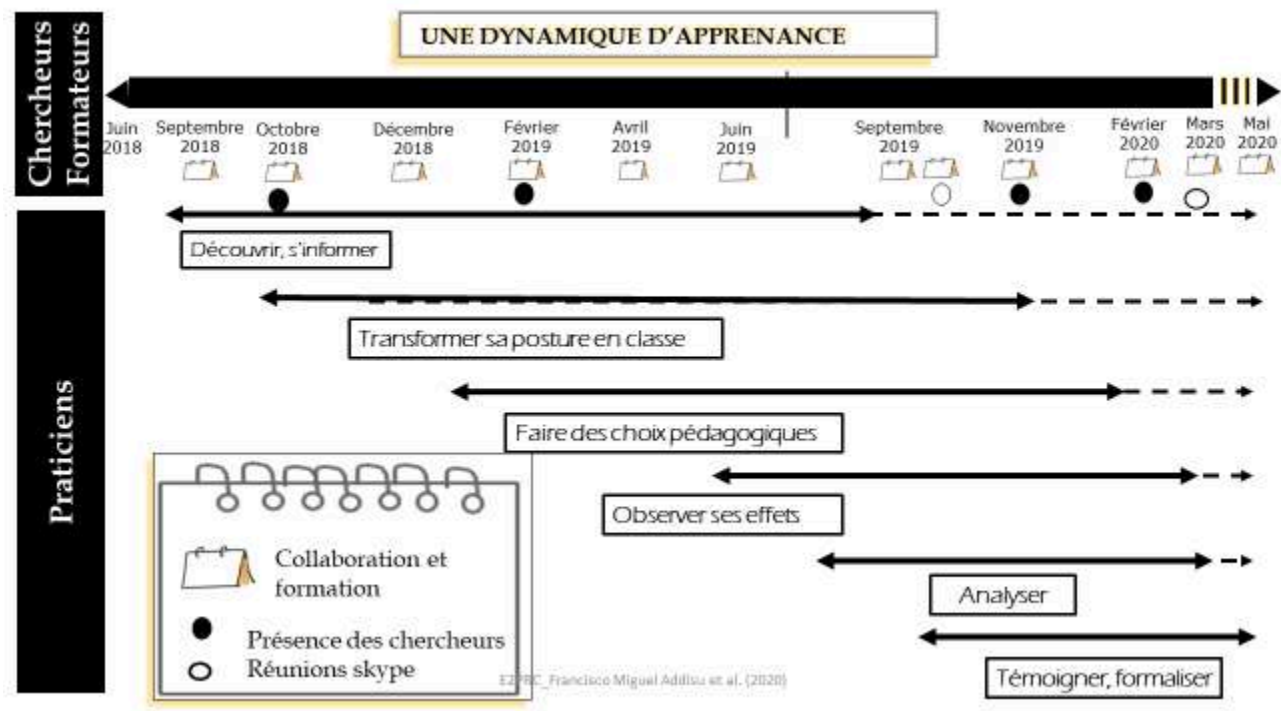


Comme le montre la figure 1 reprenant l'ensemble du projet E2PRC_Francisco, l'apprenance suppose un temps long, une durée nécessaire pour donner du sens à l'expérience partagée car « il faut ensuite faire sien le contenu de la formation, donc dégager le temps nécessaire pour poser, comprendre et résoudre les problèmes sans céder à l'impatience ambiante » (Guibert et al., $2017: 200$ ).

Outre les échanges concernant la didactique durant l'année 1, la diffusion des résultats d'un questionnaire sociolinguistique a contribué à soutenir la dynamique réflexive de l'équipe : l'étude a documenté la réalité des pratiques langagières dans l'établissement : 188 élèves de 9 à 11 ans ont répondu individuellement (plus de $90 \%$ de cette classe d'âge), ainsi que 31 enseignants et assistants français (plus de 80 \% d'entre eux).

\section{Pratiques et représentations des élèves et des enseignants de l'école SF1}

\section{Bilan d'une première enquête sociolinguistique}

L'enquête sociolinguistique montre que les profils langagiers des familles sont extrêmement diversifiés, les parcours étant souvent marqués par la mobilité : $70 \%$ des élèves répondants sont nés aux USA, $21 \%$ en France, $8 \%$ ailleurs dans le monde. Quelles que soient les langues utilisées, les élèves déclarent que près de $65 \%$ des familles sont monolingues en anglais ou en français ${ }^{6}$. Même lorsque les familles sont monolingues anglophones, la plupart des élèves déclare utiliser à la fois le français et l'anglais dans des activités personnelles, à la maison, entre amis ou dans des activités périscolaires. Le plurilinguisme des élèves est labile, il n'est pas celui des parents, ni celui des enseignants. Il est aussi largement francophone.

Si les élèves utilisent au moins deux langues au quotidien (l'anglais et le français), leurs enseignants français ont appris leur métier en mode monolingue. L'enquête à laquelle ils ont répondu au début du projet a montré qu'ils savaient quelles langues parlent leurs élèves, mais qu'ils ne voyaient pas en quoi cela concernait la didactique de l'oral. Pour eux, enseignement du français et pratiques langagières plurilingues n'étaient pas compatibles, ils avaient deux lieux différents, que les murs de la classe séparaient de fait $^{7}$. Pourtant, les enseignants volontaires qui se sont impliqués dans la RC souhaitaient dépasser ces clivages, même s'ils ne savaient pas comment. Des temps d'auto-confrontation ${ }^{8}$ ont été pour eux l'occasion d'une première prise de conscience : effectivement les langues circulaient dans la classe de français (anglais et français essentiellement, et plus rarement l'espagnol) ; mais lorsque les élèves réalisaient les tâches demandées en mode bilingue ils se cachaient presque, et donnaient l'impression de parler peu. Les praticiens expérimentés en ont peu à peu déduit qu'ils pourraient davantage favoriser les occasions d'apprendre de élèves en reconnaissant ces pratiques (valeur symbolique) et en les mobilisant comme ressources pour apprendre en français (valeur didactique). Mais la diversité des pratiques langagières les empêchait d'imaginer des séances d'oral d'une langue homogène à partir de ces pratiques hétérogènes. 


\section{Dépasser les frontières entre oral en français et pluralité langagière}

Un bilan partiel a été fait en février 2020 : la gestion de l'oral dans la classe a évolué chez ces enseignants. Ils prennent appui sur leurs connaissances des biographies plurilingues des enfants pour les valoriser, et pour choisir des modalités d'étayage qui favorisent les passages entre les langues des élèves. Dans les deux cycles, on veille à davantage expliciter, reformuler, répéter les structures du français. Les enseignants en voient des bénéfices à moyen terme, qui ont par ailleurs été pointés dans d'autres recherches (Cummins, 2014) : climat de classe plus apaisé, participation active d'enfants qui étaient quasi-muets en classe, meilleur engagement dans les tâches collaboratives ${ }^{9}$.

Peu à peu, l'oral a été envisagé par tous en tant qu'interactions susceptibles de favoriser l'appropriation des élèves, dont le plurilinguisme a pu alors être interprété en termes de ressources potentielles. Les analyses se sont poursuivies l'année 2, parallèlement à l'expérimentation de séances didactiques portant sur les biographies langagières des élèves. D'autres expérimentations ont fait suite, à l'initiative des enseignantes, qui ont porté sur des séances d'étude de la langue et d'écriture plurilingue (cycle 2), ainsi que sur l'entrée dans le langage oral (cycle 1).

La figure 1 ci-dessus représente les différentes étapes du projet du point de vue des enseignants, qui ont tous transformé leurs pratiques, mais à des rythmes différents. Si les chercheurs ont cru candidement que les résultats de l'enquête diffusée au milieu de l'année 1 suffisaient à transformer les pratiques enseignantes, c'est pourtant un ensemble de moments réflexifs qui, en se conjuguant, ont permis une évolution des gestes professionnels. La recherche-formation s'est effectivement constituée en organisation apprenante dès l'élaboration du projet par les co-pilotes.

Bien que leur rôle soit différent au sein du projet, leur préoccupation conjointe a été de veiller à la qualité des relations au sein de l'équipe, en tenant compte des contraintes de chacun : la temporalité, la disponibilité, la distance, et surtout la confiance entre partenaires qui se découvrent.

\section{Prendre soin des facteurs favorisant la collaboration}

On peut distinguer deux types d'échanges dont l'alliance est propre aux recherchesformations collaboratives. Ces deux types d'échange ont structuré le projet tout au long de l'année 1. Les échanges propres à chaque communauté ont été nombreux et récurrents; formels ou informels, ils existent dans toute formation, et dans toute recherche. Les échanges qui allient les deux communautés de pratiques sont propres aux RC (Desgagné et al., 2001) : elles ont eu lieu à deux reprises en présentiel (avec les enseignants), et à distance avec les formateurs tout au long de l'année 1.

Les modalités de travail du collectif de la recherche collaborative ont été fortement impactées par la distance : l'équipe de recherche basée en France (Université de Rouen) est à plus de 8000 kilomètres de l'école, et il y a $9 \mathrm{~h}$ de décalage horaire. Pour communiquer, des outils de visioconférences ont été mobilisés, nécessitant parfois des assistances techniques. Deux plateformes de travail ont été mises en place : la première concerne principalement les enseignants, et la seconde les chercheurs.

Les deux missions sur site des chercheurs (octobre 2018 et mars 2019) ont été vécues comme des évènements essentiels : le sentiment de collectif est né grâce aux temps de 
formation en présentiel. Ces temps forts ont été des déclencheurs de motivation de part et d'autre, probablement en partie parce que ce sont des occasions rares et donc «extra-ordinaires». La confiance entre les chercheurs et les formateurs s'est construite sur des critères de compétence didactique, mais également sur leur disponibilité, leur écoute respective, et sur leur capacité réciproque à exprimer des doutes, des difficultés, afin de co-construire une réponse. C'est selon nous ce climat de confiance qui a permis un changement de positionnement chez les quatre collaboratrices ayant facilité la collaboration sur les plans matériel, pédagogique, et scientifique (IEN, EC, DIR et CPAIEN). Mais pour ce faire, il leur a fallu tout d'abord prendre conscience du cadre hiérarchique dans lequel s'inscrivait leur action (DIR, CPAIEN) ainsi que des différences entre leurs logiques professionnelles (IEN et EC). En témoigne le bilan d'étape à la fin de l'année 1 : les échanges réunissant chercheurs et praticiens ont à la fois nourri les analyses et déstabilisé les positionnements de chacun. L'implication des chercheurs a permis en particulier de prendre conscience de relations de pouvoir au sein de l'établissement, hiérarchie qu'il fallait reconnaitre, mais aussi dépasser pour interroger sereinement les pratiques didactiques et construire les conditions de leur évolution, en liberté. Par ailleurs, la parole des praticiens rappelait sans cesse aux chercheurs la nécessité d'intégrer pleinement le réel au questionnement sociodidactique. Nous nous arrêtons sur les transformations de quatre membres qui en ont pris soin au quotidien: Elsa Rodriguez, formatrice et directrice du site (DIR), Laurence Madamour, formatrice sur plusieurs sites (CPAIEN), ainsi que les copilotes du projet (EC et IEN).

\section{Différencier son statut, sa fonction, et son rôle dans l'établissement : implication des formatrices}

\section{Diriger : DIR, de la coordination à l'implication}

DIR perçoit dès le début du projet l'atout que représente cette modalité de formation pour fédérer les enseignants et susciter la motivation, questionner les pratiques. Au début, si elle accepte de coordonner, elle estime cependant que l'accompagnement revient aux « autres » formateurs; son rôle est de faciliter l'organisation des échanges et de soutenir le projet pour obtenir le budget. Pourtant, DIR estime à la fin de l'année 1 que ses missions de coordination participent pleinement de son rôle de formation :

"Il me semble essentiel d'être déjà convaincu soi-même de la nécessité d'un tel projet tant sa mise en place va demander des ajustements et du lâcher prise dans son rôle classique de directeur. (...) Du point de vue de ma posture de directrice, au-delà de l'accompagnement traditionnel que l'on peut porter aux équipes, ce projet a nécessité parfois de ne plus être directrice mais de redevenir collègue de classe, se retrouver au même niveau de réflexion, de connaissance que les enseignants, «en se mettant à la place de » (...). Ce projet a aussi je pense permis d'assoir davantage ma crédibilité de directrice, non pas en tant que « chef» mais en tant que leader sachant mener une équipe au travers d'un processus peu commun vers des objectifs partagés par tous. » (DIR)

31 Il ne s'agit plus d'un à côté : « créer un climat de confiance » et « rassurer » contribuent pleinement à «des collectifs de co-formation capables de réfléchir ensemble sur le «bon travail» enseignant (ce qui va) susciter l'émergence de «controverses professionnelles » (Étienne, $2017: 196)$. 
Accompagner : CPAIEN, de la transmission à la problématisation du geste didactique

CPAIEN a découvert et expérimenté la modalité d'accompagnement de l'autoconfrontation. Un changement de posture a été nécessaire :

"Une de mes difficultés a été d'accepter le positionnement durant l'auto-confrontation pour bien respecter le cadre: s'émanciper du rôle " hiérarchique » de conseillère pédagogique pour mieux guider la parole de l'enseignant vers un discours sur l'activité réelle sans que celui-ci soit sur la défensive ou la justification.» (CPAIEN)

Dès les premiers accompagnements, elle relève "les grands changements des deux collègues dans leur prise en compte du contexte et donc dans leur pratique quotidienne, comme "une révélation. ». Enfin, elle souligne que l'apprenance n'est possible que si on s'inscrit soi-même dans cette logique avec d'autres membres du projet, les chercheurs en particulier :

"Le dialogue qui s'installe [avec les enseignants] où mon rôle est d'amener l'enseignant à parler, comprendre, analyser son activité sans jamais essayer de l'influencer, a été dans un premier temps très déstabilisant et très frustrant. Le fait de débriefer ensuite avec les collègues [les chercheurs en particulier] sur nos ressentis a été un élément essentiel dans la prise de confiance, dans les liens créés. » (CPAIEN)

36 À l'issue de l'année 1, CPAIEN et DIR se sont engagées dans une démarche de construction d'outils avec les enseignants.

\section{Différencier son statut, ses fonctions, et son rôle dans sa communauté de pratique : implication des co-pilotes}

\section{Chercher : EC, de la problématisation à l'accompagnement praxéologique}

En s'engageant dans une recherche qui s'appuie sur les savoirs savants et sur les savoirs pratiques sans les hiérarchiser, EC devait aussi accepter d'oser répondre aux questionnements des praticiens avant même que la recherche ait réellement abouti à des résultats fiables. Cela a été le cas de tous les chercheurs du projet, à des moments différents de la collaboration, et principalement lorsque les rencontres se faisaient dans le quotidien des classes, ou dans la connivence des entretiens.

Ce faisant, les échanges ont orienté les choix des analyses de données et les problématisations qu'elles sous-tendent: dès la fin de l'année 1, le travail de documentation des pratiques langagières (analyse sociolinguistique) s'est réorganisé en fonction de la réalité des questionnements pratiques des enseignants et formateurs (orientation didactique) :

39 "Si les freins des enseignants existent, ils sont peut-être pertinents, je ne peux donc les évacuer au nom d'une doxa de chercheur : comment en tenir compte? comment les accompagner ? (...) La collaboration autorise à "lâcher prise » sur les objectifs du projet : en fait le projet a des objectifs multiples que personne ne maitrise complètement, mais la collaboration est fondée sur la croyance de chacun qu'en "servant » les objectifs de l'autre, le travail commun servira aussi son objectif: interdépendance bénéfique ». (EC)

Six mois plus tard, EC explique que le projet est positif pour deux raisons, même s'il lui faut faire le deuil d'un étayage langagier idéal, auquel aucun contexte de classe ne peut prétendre. D'une part, les enseignants sont désormais capables de choisir et de décrire 
des gestes professionnels pertinents en s'appuyant sur des analyses didactiques outillées par les recherches en sociolinguistique et didactique du plurilinguisme: le projet a permis que les enseignants fassent désormais « le choix et le développement de pratiques de classe qu'ils considèrent comme innovantes, dont ils n'auraient pas eu l'idée, l'envie, la nécessité d'exploiter sans la recherche collaborative" (IEN). D'autre part, de nouvelles connaissances sont aujourd'hui disponibles pour comprendre comment il est possible de s'appuyer sur les ressources plurilingues des élèves pour favoriser leur appropriation en français. La recherche confirme que l'oral, ici, est moins une question de genre qu'une question de passage entre différentes modalités langagières, qui contribuent toutes à la dynamique d'appropriation :

Contrairement à ce qu'on aurait pu penser à priori, ce n'est pas le plurilinguisme initial des enfants qui fait la différence dans la réussite des tâches proposées, pas plus que l'instauration d'un bilinguisme scolaire, mais plutôt les modes de gestion des répertoires (individuels et collectifs) et l'aménagement d'un type de cadre didactique et de culture métalinguistique qui valorise l'ouverture et la diversité, le rapprochement, la comparaison, la collaboration et une réflexion métalinguistique. (Castellotti \& Moore, $2005:$ 128)

\section{Décider : IEN, de l'autorité à la médiation}

41 Le processus d'apprenance supposait que l'IEN, représentante de l'institution et garante de la qualité des enseignements, accepte à son tour un " lâcher - prise » : faire confiance à la capacité des enseignants de construire par eux-mêmes des modalités de travail adaptées à leurs élèves grâce à l'accompagnement mis en œuvre. Très vite, ce rôle de pilote plutôt effacé dans le quotidien du projet a évolué vers une médiation nécessaire entre les différents acteurs (CPAIEN, DIR, EC) pour favoriser, malgré la distance, la relation de confiance nécessaire à la collaboration. Il fallait pour cela renvoyer à chaque acteur une perception globale de la dynamique du projet, ce qu'aucun d'entre eux ne pouvait percevoir lors de l'année 1 :

42 "La recherche collaborative est un moyen, qui sert aux différents acteurs de façon complémentaire, chacun gardant des objectifs propres. Il y a une interdépendance: les chercheurs ont besoin du retour des praticiens et de leurs traces, les praticiens ont besoin de l'analyse de la recherche et des explications des chercheurs pour s'appuyer sur des concepts et données qu'ils n'auraient pas sans la recherche collaborative » (IEN)

43 Cela a nécessité une clarification des représentations du rôle de chacun par l'IEN, développant un point de vue qui rappelle celui des passeurs en éducation :

Le passeur/formateur aborde de façon pragmatique les questions vives issues $d u$ terrain en s'adossant à la recherche, pour construire des compétences professionnelles efficientes. (...) [il prend] au sérieux les savoirs et les connaissances spécifiques qui seraient constitutifs d'une compétence de "passeur", non réductibles à un simple voisinage organisé entre praticiens et scientifiques (Gaussel et al., $2017: 37$ )

44 Ce rôle de médiation et de passeur en éducation nous apparait aujourd'hui lui aussi comme constitutif de la dynamique de formation dans cette recherche: par son pilotage, l'IEN doit pouvoir croiser les logiques pratiques et scientifiques au bénéfice des établissements scolaires dont il a la responsabilité :

"L'année a beaucoup enrichi ma compréhension du milieu de la recherche universitaire en éducation, apporte une meilleure compréhension du rôle du chercheur. Si le volet formation est 
trop présent pour une recherche collaborative, finalement peut-être que l'on fait en réalité une «Recherche-Formation collaborative »? (IEN)

\section{Conclusion}

expérience de recherche-formation collaborative met à jour la complexité d'une dynamique qui s'inscrit dans le réel. Elle s'appuie sur tous les acteurs-formateurs, qui conjuguent tous à leur manière implication, pilotage, accompagnement sur le terrain, problématisation théorique orientée par la pratique. Par l'objet qu'elle interroge (la didactique de l'oral en contexte plurilingue), la RC E2PRC_Francisco a dû prendre acte des représentations monolingues des enseignants, et développer une logique d'apprenance qui les intégrait pleinement, sous peine de ne rien pouvoir construire. Le temps long permet à chacun d'accepter l'autre dans ses différences, et d'accepter de considérer les écarts comme des lieux de progression potentielle.

Alors que la recherche E2PRC_Francisco s'attache à travailler le plurilingue, les enseignants n'opposent plus didactique de l'oral et plurilinguisme dans leurs pratiques, les formateurs savent qu'ils n'ont pas tout à transmettre, les chercheurs acceptent de risquer leurs convictions à l'aune des pratiques.

Tous se sont trouvés en situation de questionner leur identité professionnelle de façon également interculturelle, afin de créer des ponts entre recherche et éducation. En bousculant les cadres interprétatifs de chaque culture professionnelle («évidences pédagogiques, hiérarchiques, ou scientifiques »), la RC a permis la naissance d'une écoute active de chacun, et ensuite de nouveaux choix professionnels au bénéfice des élèves. Ces choix s'appuient sur une nouvelle « évidence " pour la didactique de l'oral : malgré la tension entre deux modèles interactionnels (monolingue ou plurilingue) la pluralité des usages langagiers sert l'appropriation du français en contexte multilingue. Les choix pédagogiques qui découlent de cette évidence demeurent cependant le fait des praticiens.

La recherche collaborative présente aussi ses limites pour les praticiens comme pour les chercheurs c'est une démarche exigeante en temps et couteuse en moyens. Il a fallu prioriser ce projet au détriment d'autres actions ou thématiques, il a fallu apprendre à utiliser de nouveaux outils, accepter des temporalités différentes propres à chaque communauté engagée. Personne ne maitrise l'affaire, personne ne décide pour l'autre, que l'on ne comprend jamais tout à fait, sauf à vouloir ensemble com-prendre (au sens de «prendre ensemble »). Un tel dispositif suppose donc un cahier des charges souple, mais construit collectivement dès le début du projet. Par ailleurs, si les RC nous paraissent à même de favoriser de nouvelles connaissances sociodidactiques et de contribuer au développement de pratiques d'enseignement spécifiques à un contexte scolaire, l'évaluation de la progression des élèves est un volet de la problématique sur lequel praticiens et chercheurs poursuivent encore leur collaboration.

Schön, D. A. (1984). The Reflective Practitioner : How Professionals Think In Action. New York : Basic Books. 


\section{BIBLIOGRAPHIE}

AEFE (2015). Pour une éducation plurilingue, la politique des langues de l'AEFE. Les Cahiers de l'AEFE, AEFE.

Anadon, M. (2013). « Recherche participative ». In Casillo, I., Barbier, R., Blondiaux, L., Chateauraynaud, F., Fourniau, J-M., Lefebvre, R., Neveu, C. et Salles D. (dir.), Dictionnaire critique et interdisciplinaire de la participation, Paris, GIS Démocratie et Participation.

Beaumont, S., Moore, D. (2020). « Plurilinguisme et formation des enseignants de l'éducation nationale en Amérique du Nord. Une recherche-action-formation pour le développement d'outils et pratiques innovantes ». Recherches et Application $n^{\circ}$ 67, 30-40. Site du laboratoire du plurilinguisme : https://sites.google.com/view/aefe-plurilinguisme/.

Bucheton, D., Soulé Y. (2009). «Les gestes professionnels et le jeu des postures de l'enseignant dans la classe : un multi-agenda de préoccupations enchâssées ", Éducation et didactique, vol 3 $\mathrm{n}^{\circ} 3,29-48$.

Carré, P. (2005). L'apprenance, Paris : Dunod.

Castellotti, V. (2013). «L'articulation recherche-intervention en DDL : comment (ne pas) en sortir?». In Beacco, J.-C. (dirs.), Ethique et politique en didactique des langues. Autour de la notion de responsabilité. Paris : Didier, 74-98.

Castellotti, V., Moore D., (2005). «Répertoires pluriels, culture métalinguistique et usages d'appropriation ». In Beacco, J.-C., Chiss, J.-L., Cicurel, F., Véronique, D. (dirs.). Les cultures éducatives et linguistiques dans l'enseignement des langues, Paris : PUF, 107-132.

Cicurel, F. (2011). «De l'analyse des interactions en classe de langue à l'agir professoral : une recherche entre linguistique interactionnelle, didactique et théories de l'action ». Pratiques, $149-150,41-55$

Clot, Y., Faïta, D., Fernandez, G., Scheller, L. (2001). « Entretiens en auto-confrontation croisée : une méthode en clinique de l'activité ». Education Permanente, 146(1), 17-25.

Cummins, J. (2014). «L'éducation bilingue : qu'avons-nous appris de cinquante ans de recherche ?». In Nocus, I., Vernaudon, J., Paia, M. (éds.), L'école plurilingue en Outre-mer Apprendre plusieurs langues, plusieurs langues pour apprendre. Rennes : Presses universitaires de Rennes, 41-64.

Desgagné, S., Bednarz, N., Lebuis, P., Poirier, L. \& Couture, C. (2001). « L'approche collaborative de recherche en éducation : un rapport nouveau à établir entre recherche et formation ». Revue des sciences de l'éducation, 27 (1), 33-64.

Dolz, J., Schneuwly, B. (1998). Pour un enseignement de l'oral. Les genres formels à l'école. Paris : E.S.F.

Etienne, R. (2017). « Rôle de l'analyse dans une formation en alternance : faire avec la complexité et accompagner le changement ». In Desjardins J., Beckers J., Guibert P., Maulini O. (dirs.). Comment changent les formations enseignantes? Bruxelles, De Boeck Supérieur, 177-196.

Gajo, L. (2009). «Politiques éducatives et enjeux socio-didactiques : l'enseignement bilingue francophone et ses modèles ». Glottopol, 13, 16-27.

Gaussel, M., Gibert, A., Joubaire, C., Rey, O. (2017). « Quelles définitions du passeur en éducation? ». Revue française de pédagogie, 201(4), 35-39. 
Guibert, P., Maulini, O., Desjardins, J., Beckers, J. (2017). « Des facteurs de changement aux stratégies des acteurs ». In Desjardins, J. Beckers, P. Guibert, O. Maulini. (dirs.) Comment changent les formations enseignantes? Bruxelles : De Boeck Supérieur, 197-202.

Halté, J.-F. (2005). « Intégrer l'oral : Pour une didactique de l'activité langagière ». In Halté, J.-F., Rispail, M. (dir.), L'Oral dans la classe, Compétences, enseignement, activités. Paris : L'Harmattan, 11-31.

Lafontaine, L. (2013). Enseigner l'oral, c'est possible ! : 18 ateliers formatifs clés en main. Quebec : Chenelière Education.

Morrissette, J. (2013). « Recherche-action et recherche collaborative : quel rapport aux savoirs et à la production de savoirs ?». Nouvelles pratiques sociales, 25(2), 35-49.

Nonnon, E. (2011). « L'histoire de la didactique de l'oral, un observatoire de questions vives de la didactique du français ». Pratiques, 184-206.

Nussbaum, L. (2014). « Le parler plurilingue comme ressource d'apprentissage ». Tréma, 42, 76 -

85.

Pégaz-Paquet, A., Cadet, L. (2016). « Prendre/apprendre la parole : l'oral à l'école primaire dans les textes officiels ». Le français aujourd'hui, 195(4), 9-22.

\section{NOTES}

1. Afin d'accompagner les enseignants titulaires de l'éducation nationale et les recrutés locaux à garantir une excellence éducative, 16 inspecteurs.trices de l'éducation nationale sont détaché.e.s auprès de l'AEFE pour les écoles primaires dans le monde. IEN, initiatrice du projet, est responsable de la politique de formation auprès des 54 établissements de la zone Amérique du Nord.

2. 9 enseignants l'année 1,1 a quitté l'établissement et 2 sont arrivés l'année 2.

3. L'équipe de chercheurs rouennais est composée de quatre membres ayant une expertise complémentaire, au sein du laboratoire Dynamiques du langage in situ. Ils ont chacun contribué à la formation lors des différentes missions à San Francisco, et ont conjointement participé à l'analyse des données sociolinguistiques et didactiques.

4. Le processus de co-écriture que nous avons adopté est le suivant: après des échanges nombreux entre les auteurs et avec les formateurs engagés dans le dispositif, le premier auteur de chaque partie a soumis son texte au second auteur, les échanges se sont poursuivis, l'écriture s'est peu à peu stabilisée et la dernière partie a été écrite conjointement. Les expertises externes de la première version de ce texte ont beaucoup aidé à clarifier le texte dans cette version révisée.

5. Pour ce qui concerne le geste professionnel, les travaux sur le multi-agenda ont été proposés mais finalement peu investis par les enseignants (Bucheton et Soulé, 2009). Pour ce qui concerne l'enseignement de l'oral, il a d'abord été choisi de proposer des ressources disponibles sur le portail du ministère Eduscol parce qu'elles paraissaient légitimes aux praticiens tout en étant suffisamment problématisantes pour les chercheurs (voir par exemple: https:// eduscol.education.fr/cid103155/francais-cycle-langage-oral.html). Puis les ressources ont été diversifiées (voir par exemple la très éclairante conférence de Sylvie Plane : «L'oral un objet multidimensionnel » en 2019: http://centre-alain-savary.ens-lyon.fr/CAS/education-auplurilinguisme/conference-sylvie-plane).

6. La plupart des autres familles utilise conjointement ces deux langues (22\%). Les autres langues mentionnées comme participant pleinement aux interactions familiales sont l'espagnol, le 
japonais, l'arabe, le russe, l'italien... mais une trentaine de langues différentes sont mentionnées en tant que langues parlées avec divers membres de la famille (y compris dans les familles monolingues).

7. Une seconde enquête par entretiens est en cours, dirigée par Christel Troncy.

8. L'auto-confrontation fait référence à la théorie de l'activité (Clot et al. 2001). Dans un premier temps, l'enseignant met à jour des schèmes didactiques structurant son activité en analysant sa pratique à partir de vidéos. Il conscientise ainsi ses actes et l'orientation des interactions au sein de la classe. Dans un second temps, chercheurs et enseignants croisent leurs analyses et les enrichissent par une grille de lecture théorique portant à la fois sur les postures d'étayage au sein des interactions (Bucheton et Soulé, 2009) et sur les postures interactionnelles en situation exolingue (Gajo, 2009).

9. Une étude critériée contextualisée est en cours. Des productions pédagogiques contextualisées seront élaborées par les enseignants, en particulier pour leurs collègues nouvellement arrivés dans l'établissement. Si ces outils s'avèrent transposables, ils pourront être diffusés en 2021 sur le site du laboratoire du plurilinguisme Enseigner en contexte plurilingue - Laboratoire d'analyse de pratiques et de production de ressources (AEFE) issu d'une recherche-formation co-pilotée par Sophie Beaumont et Danièle Moore au même moment que cette recherche, mais construite sous la forme de modules de formation réunissant une vingtaine d'autres enseignants volontaires sur toute la zone Amérique du Nord (Beaumont \& Moore, 2020).

\section{RÉSUMÉS}

Dans le champ des recherches collaboratives en didactique des langues, les acteurs des projets de recherche-formation postulent que la collaboration répond aux besoins des praticiens et des chercheurs. Or les résultats attendus ne relèvent pas de la même épistémologie (épistémologie pratique et/ou savante). Pourtant, si la collaboration est possible, nous postulons que c'est parce que des leviers existent, qui ne sont pas uniquement de l'ordre du relationnel, de «l'heureuse rencontre ", ou même d'une éthique partagée qui définirait plus spécifiquement les RC. Le postulat de départ est que la collaboration est possible, et souhaitable, au bénéfice de tous, $\mathrm{y}$ compris des élèves. Nous en discuterons à partir d'une expérience de recherche-formation collaborative dans le champ de la didactique des langues.

Le projet École, Parole de l'élève et Plurilinguisme: Recherche Collaborative au lycée français de San Francisco - AEFE, USA (E2PRC_Francisco) prend pour objet la didactique de l'oral en contexte plurilingue et réunit 12 enseignants, 2 formatrices, 4 chercheurs, 1 inspectrice. Nous nous intéresserons plus spécifiquement à l'analyse du processus de collaboration entre chercheurs et formateurs par les deux voix des co-pilotes du projet. Après avoir présenté le contexte de la recherche, nous montrerons comment les données sociolinguistiques ont servi une démarche partagée d'apprenance. Nous nous arrêterons sur les transformations et déplacements professionnels qu'un tel dispositif a initié pour les formateurs et les co-pilotes.

In the field of collaborative research in the didactic of languages, the actors of research-training projects postulate that collaboration meets the needs of both practitioners and researchers. Yet as it happens, the expected results do not reveal the same epistemology (practical and/or academic). However, if collaboration is possible, our premise is that it is thanks to the existence of triggers, which do not only include people skills, fortuitous encounters, or even shared ethics 
specific to collaborative researches. The basic principle is that collaboration is possible and even wanted as a benefit for each participant, pupils included. We will discuss from a collaborative research-training project in the area of the didactic of languages.

The project 'Schools, Pupils' Languages and Plurilingualism: a collaborative research in the International French School of San Francisco - AEFE, USA (E2PRC_Francisco)' is dealing with the didactic of oral language in a plurilingual context and gathers 9 teachers, 2 trainers for teachers, 4 researchers, 1 French inspector of education. We will more specifically focus on how researchers and trainers collaborate, through the double point of view of the co-pilots of the project who shared the writing. The article starts with a description of the context, and we will then demonstrate how the sociolinguistic data helped to achieve a shared approach of self-directed learning. We will finally detail the transformations and professional consequences initiated by such a conceptual framework for the trainers as well as for the co-pilots.

\section{INDEX}

Mots-clés : plurilinguisme, réflexivité, AEFE, recherche collaborative, sociodidactique

Keywords : plurilingualism, reflexive practice, AEFE, collaborative research, socio-didactics

\section{AUTEURS}

\section{VÉRONIQUE MIGUEL-ADDISU}

Université de Rouen, France

Mes travaux se situent entre sociolinguistique et didactique, et visent à contribuer à une meilleure connaissance des pratiques langagières des locuteurs plurilingues en situation de contact de langues. À l'école, je cherche à comprendre comment les élèves plurilingues s'approprient la langue de scolarisation en situation éducative immersive en France et à l'étranger, et à construire une approche plurielle de la didactique du français, à visée inclusive, altéritaire. veronique.migueladdisu[at]univ-rouen.fr

\section{SOPHIE BEAUMONT}

\section{AEFE, France}

Mon métier d'inspectrice de l'éducation nationale détachée auprès des établissements français AEFE m'a amenée à m'interroger sur les spécificités de l'apprentissage du français en contexte anglophone. Mes questionnements partent des écoles visitées et des échanges avec les acteurs, qui sont confrontés à des problématiques spécifiques au plurilinguisme et font l'objet de deux recherches formation collaboratives, E2PRC_Francisco (cf. ci-dessous) et un dispositif intitulé laboratoire AEFE d'analyse et de production de ressources du plurilinguisme.

ien.an[at]aefe.fr 